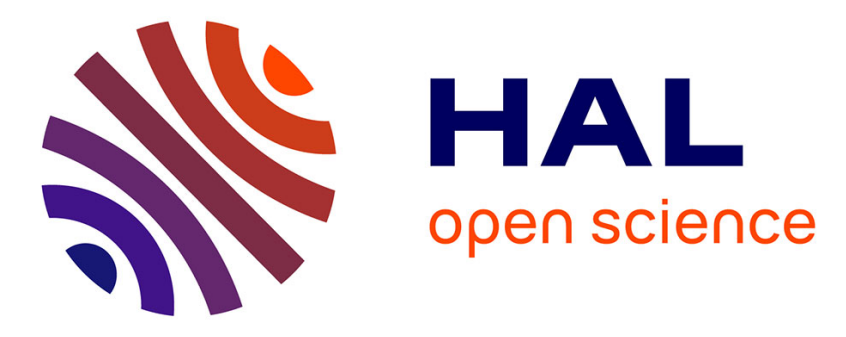

\title{
Diamagnetically trapped arrays of living cells above micromagnets
}

Paul Kauffmann, Ammara Ith, Daniel O’Brien, Victor Gaude, Florian Boué,

Stéphanie Combe, Franz Bruckert, Béatrice Schaack, Nora Dempsey, Vincent

Haguet, et al.

\section{To cite this version:}

Paul Kauffmann, Ammara Ith, Daniel O'Brien, Victor Gaude, Florian Boué, et al.. Diamagnetically trapped arrays of living cells above micromagnets. Lab on a Chip, 2011, 11 (18), pp.3153-3161. 10.1039/c1lc20232d . hal-01067514

\section{HAL Id: hal-01067514 https://hal.science/hal-01067514}

Submitted on 24 Oct 2017

HAL is a multi-disciplinary open access archive for the deposit and dissemination of scientific research documents, whether they are published or not. The documents may come from teaching and research institutions in France or abroad, or from public or private research centers.
L'archive ouverte pluridisciplinaire HAL, est destinée au dépôt et à la diffusion de documents scientifiques de niveau recherche, publiés ou non, émanant des établissements d'enseignement et de recherche français ou étrangers, des laboratoires publics ou privés. 


\title{
Diamagnetically trapped arrays of living cells above micromagnets $\dagger$
}

\author{
Paul Kauffmann, ${ }^{* a b}$ Ammara Ith, ${ }^{a}$ Daniel O'Brien, ${ }^{c}$ Victor Gaude, ${ }^{a}$ Florian Boué, ${ }^{a}$ Stéphanie Combe, ${ }^{b}$ \\ Franz Bruckert, ${ }^{d}$ Béatrice Schaack, ${ }^{b}$ Nora M. Dempsey, ${ }^{c}$ Vincent Haguet $^{b}$ and Gilbert Reyne ${ }^{a}$
}

Cell arrays are of foremost importance for many applications in pharmaceutical research or fundamental biology. Although arraying techniques have been widely investigated for adherent cells, organization of cells in suspension has been rarely considered. The arraying of non-adherent cells using the diamagnetic repulsive force is presented. A planar arrangement of Jurkat cells is achieved at the microscale above high quality microfabricated permanent magnets with remanent magnetization of $J_{\mathrm{r}} \approx 1 \mathrm{~T}$, in the presence of a paramagnetic contrast agent. The cytotoxicity of three Gd based contrast agents, Gd-DOTA, Gd-BOPTA and Gd-HP-DO3A, is studied. Among them, Gd-HP-DO3A appears to be the most biocompatible toward Jurkat cells. In close agreement with analytical simulations, diamagnetically 'suspended' cells have been successfully arrayed above square and honeycomb-like micromagnet arrays, which act as a "diamagnetophobic" surface. Living cell trapping is achieved in a simple manner using concentrations of Gd-HP-DO3A as low as $1.5 \mathrm{mM}$.

\section{Introduction}

Single cell experiments should have a large impact on biomedical studies in the years ahead. When inherent heterogeneity (e.g. stochastic gene expression) and variable sensitivity of cells to the environment are investigated, reproducible cell arraying is the first step to provide statistics on cells. ${ }^{1}$ After the planar distribution of hundreds or thousands of cells onto the cell array device, a multi-parametric characterization of every cell using fluorescence microscopy can be carried out to supply a High Content Analysis (HCA) of the cell population. Large bidimensional arrangements of cells are advantageous for characterizing small quantities of cells according to their properties, e.g. for identifying rare tumorous cells or stem cells among a wide population. Furthermore, cell arrays allow the monitoring of the behaviour of individual cells for minutes, hours or even days.

${ }^{a}$ Grenoble Electrical Engineering Laboratory (G2Elab), UMR 5269 (Grenoble-INP, UJF, CNRS), BP 46, 38402 Saint Martin d'Hères

Cedex, France. E-mail: paul.kauffmann@g2elab.grenoble-inp.fr; Fax:+33 (0)4 768263 00; Tel: +33 (0)456529431

${ }^{\mathrm{b}}$ Commissariat à l'Energie Atomique et aux Energies Alternatives (CEA), BGE, U1038 (CEA, Inserm, UJF), 17 rue des Martyrs, 38054 Grenoble Cedex 9, France. E-mail: vincent.haguet@cea.fr; Fax: +33 (0)4 387859 17; Tel: +33 (0)438782386

'Institut Néel CNRS/UJF, 25 avenue des Martyrs, BP 166, 38042 Grenoble Cedex 9, France. E-mail: nora.dempsey@grenoble.cnrs.fr; Fax: +33 (0)4 7688 11 91; Tel: +33(0)476 887435

dLaboratoire des Matériaux et du Génie Physique (LMGP), UMR 5628 (CNRS, Grenoble-INP), Grenoble INP Minatec, 3 parvis Louis Néel, BP 257, 38016 Grenoble, France. E-mail: franz.bruckert@grenoble-inp.fr; Fax: +33 (0)4 565293 01; Tel: +33 (0)43878 5419
Other applications of living cell arrays include cell observation based diagnostics, identification of the genetic role in diseases, study of communication between cells, drug screening, cell therapy, cell reprogramming and using this first level of organization as a starting point for tissue engineering.

Current arraying techniques strongly depend on whether cells can adhere to a substrate or not. Many efficient techniques have been investigated for adherent cells such as protein micropatterns for local cell adhesion ${ }^{2,3}$ or microfluidic isolation of a single cell. ${ }^{4}$ Cells have also been labelled by magnetic micro- or nano-particles either attached specifically to their membrane (immunolabelling) or internalized. As a result, they are strongly attracted towards the edge of the magnetic patterns. ${ }^{5-7}$

On the other hand, arraying techniques of cells in suspension have scarcely been investigated although such cells are widespread in nature and in tissue culture laboratories. The most obvious example is circulating cells, i.e. blood cells, which travel through the arteries and veins. Another example is the Circulating Tumor Cell (CTC), a cancerous cell detached from a primary tumor, which flows through the blood in search of a new implantation. Due to human manipulation, adherent cells can also be temporarily in suspension, e.g. sedimenting cells during cell seeding or adherent cells returned to the suspension after gentle scraping or biochemical detachment using trypsin or proteinase K. Furthermore, suspension-adapted cell cultures have been developed from adherent cell lines for the biotechnological production of recombinant proteins in bioreactors. Apoptotic cells can also detach during the mortality mechanism.

The most common way to array cells in suspension exploits topological microwells formed on the surface of a substrate. This technique has been optimized for single cell trapping. ${ }^{8}$ It can also 
be used to retain cells during rinsing steps. ${ }^{9}$ Topological microwells have been modified to retrieve target cells from the array and have been applied to study B lymphocytes. ${ }^{10}$ More recently, large enough wells have been manufactured for the proliferation of cells in a microfluidic environment. ${ }^{11}$ However, the direct contact of non-adherent cells such as lymphocytes with surfaces is suspected to modify the behaviour of the cells. ${ }^{12,13}$ In the arraying process of cells in suspension, the difficulty to control the spatial distribution of cells without tethering ${ }^{9}$ is a technology issue which needs to be tackled.

Several contactless handling techniques have been developed for micron-sized particle trapping. ${ }^{14-17}$ Most of them rely on gradient forces proportional to " $A$.d $A$ ", where $A$ represents a given field (e.g. electromagnetic, electric, magnetic, acoustic, ...) and $\mathrm{d} A$ its gradient. Most of the time, these handling techniques are exploited as label-free techniques. Contactless manipulations are based on the contrast of physical characteristics between the buffer (i.e. cell medium) and the microparticle (i.e. cell). This Archimedes principle can be optical, electrical, mechanical or magnetic. The three former repulsive forces have been previously used to trap cells in potential wells for the arraying of cells. Discovered by Ashkin in 1970, ${ }^{14}$ optical manipulation has been improved to perform 3D holographic optical traps. ${ }^{15}$ Dielectrophoretic forces have also been largely investigated for cell arraying using integrated electrodes. ${ }^{16}$ Acoustic tweezers have been employed to trap red blood cells in acoustic nodes. ${ }^{17}$ Nevertheless, these techniques suffer from weak throughput, Joule heating or set-up complexity.

Among the contactless trapping forces, diamagnetism is the least investigated. Achieving diamagnetic trapping of objects having rather low diamagnetic susceptibilities such as cells requires both strong magnetic fields and strong field gradients. ${ }^{18,19}$ At the microscale, these two conditions can be fulfilled ${ }^{20}$ thanks to the successful integration of high quality $\mathrm{NdFeB}$ micromagnets on silicon wafers. ${ }^{21,22}$ The present work demonstrates contactless arraying of small quantities of Jurkat cells on two different $\mathrm{NdFeB}$ micromagnet array devices. Basics on the diamagnetic force obtained with permanent micromagnets are first presented. Micromagnet fabrication and cell population preparation are then detailed. Results on contrast agent (CA) cytotoxicity and successful cell trapping are finally presented.

\section{Theory}

Submitted to a non-uniform magnetic field $B$, a particle (e.g. a cell) suspended in a buffer undergoes an apparent repulsive volume force $F$ :

$$
\vec{F}=\frac{\chi_{\mathrm{p}}-\chi_{\mathrm{m}}}{2 \mu_{0}}(\vec{B} \cdot \vec{\nabla}) \vec{B}
$$

where $\chi_{\mathrm{p}}$ and $\chi_{\mathrm{m}}$ are the susceptibilities of the particle and of the buffer medium, respectively, and $\mu_{0}$ is the vacuum permeability. Two main points should be clarified about this equation:

The first is that, similar to the Archimedes principle, the diamagnetic apparent force is the resultant of the magnetic buoyancy force and the diamagnetic repulsive force. As diamagnetic materials and especially biological materials (cells, bacteria, ...) have a susceptibility close to water $\left(-9 \times 10^{-6} \mathrm{SI}\right)$, the susceptibility contrast is close to zero. For this reason, a paramagnetic contrast agent (CA) is added to the medium in order to create a susceptibility contrast which produces a repulsive diamagnetic force. Hence, the buffer needs to be less diamagnetic (or more paramagnetic) than the cells.

Secondly, the magnetic force also depends on the magnetic field gradient. For a long time, the main way to obtain forces efficient enough to compensate for gravity has been to generate high magnetic fields using superconducting and Bitter coils. ${ }^{23-25}$ Another method is to increase the field gradient. By decreasing the size of a magnet by a factor $k$, the generated volume force can be increased by the same factor. ${ }^{20}$ The dimensions of magnetic systems have been decreased down to the centimetre and the millimetre scales so as to compensate the weight of diamagnetic particles and thus achieve diamagnetic trapping. ${ }^{18,19,26,27}$ However, fabrication of high quality, MEMS compatible permanent magnets has been a bottleneck for the successful scaling down of the system in the drive towards everincreasing field gradients. Recent breakthroughs in magnetic thick film deposition ${ }^{21,22}$ have led to the creation of both a strong remanence $J_{\mathrm{r}}(1 \mathrm{~T})$ and field gradients as high as $10^{6}$ $\mathrm{T} \mathrm{m}^{-1}$ (ref. 28).

\section{Materials and methods}

\section{Micro-fabrication process}

To obtain strong magnetic gradients, a $\mathrm{NdFeB}$ magnetic layer was deposited onto a micro-patterned silicon wafer (Fig. 1). Photolithography was performed on a $100 \mathrm{~nm}$ thick aluminium layer sputtered onto the bulk Si wafer (Fig. 1A). The Al layer was chemically etched (Fig. 1B) so as to provide a hard mask far more resistant to Deep Reactive Ion Etching (DRIE) than a standard photoresist mask. After the removal of the photoresist, a $90 \mu \mathrm{m}$ deep anisotropic silicon etch was performed using a Multiplex ICP (inductive coupled plasma) chamber from Surface Technology Systems (Newport, United Kingdom) (Fig. 1C). A Bosch process was used with a $\mathrm{C}_{4} \mathrm{~F}_{8}$ plasma for wall passivation cycles and a $\mathrm{SF}_{6} / \mathrm{O}_{2}$ plasma for etching cycles. The $\mathrm{Al}$ layer was then removed and the wafer cleaned of organic particles and native oxide using successive baths of $\mathrm{HF}: \mathrm{H}_{2} \mathrm{O}, \mathrm{H}_{2} \mathrm{SO}_{4}: \mathrm{H}_{2} \mathrm{O}_{2}$, $\mathrm{HF}: \mathrm{H}_{2} \mathrm{O}$ and finally $\mathrm{H}_{2} \mathrm{O} .100 \mathrm{~nm}$ of low thermal silicon oxide (Fig. 1D) and $100 \mathrm{~nm}$ of tantalum (Fig. 1E) were deposited by

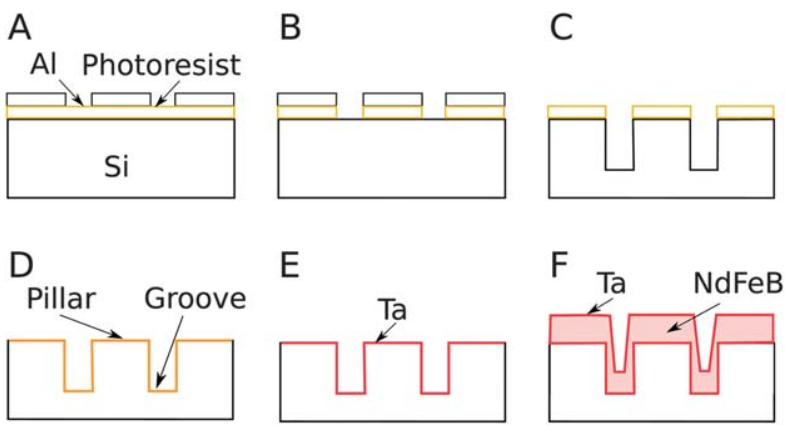

Fig. 1 Process steps of $\mathrm{NdFeB}$ magnet microfabrication. (A) Photolithography on an Al layer. (B) Chemical etching of Al. (C) DRIE. (D) Photoresist and $\mathrm{Al}$ removal. A $100 \mathrm{~nm}$ thick $\mathrm{SiO}_{2}$ layer is deposited by PECVD. (E) Sputtering of $100 \mathrm{~nm}$ thick Ta layer. (F) Sputtering of $30 \mu \mathrm{m}$ thick NdFeB and $500 \mathrm{~nm}$ Ta layers. 
Plasma Enhanced Chemical Vapour Deposition (PECVD) and by sputtering, respectively. The $\mathrm{SiO}_{2}$ layer serves as a reaction barrier between $\mathrm{Si}$ and $\mathrm{Ta}$, while the Ta layer serves as a reaction barrier between $\mathrm{SiO}_{2}$ and the NdFeB layers.

A $30 \mu \mathrm{m}$ thick $\mathrm{NdFeB}$ film was then deposited via triode dc sputtering at a rate of approximately $15 \mu \mathrm{m} \mathrm{h}^{-1}$ (Fig. 1F). The $\mathrm{NdFeB}$ layer was deposited at a temperature of $400{ }^{\circ} \mathrm{C}$ and was then capped with a $100 \mathrm{~nm}$ layer of Ta. The as-deposited film was amorphous, and the high anisotropy $\mathrm{Nd}_{2} \mathrm{Fe}_{14} \mathrm{~B}$ phase was crystallized during a post-deposition anneal $\left(750{ }^{\circ} \mathrm{C}\right.$ for 10 minutes). After wafer dicing into chips of $8 \times 8 \mathrm{~mm}$, a layer of $500 \mathrm{~nm}$ of Ta was sputtered onto the deposit to protect the sidewalls (Fig. 1F). The resulting micromagnets had a coercivity $\mu_{0} H_{\mathrm{c}}$ of $1.6 \mathrm{~T}$ and an out of plane remanent polarisation $J_{\mathrm{r}}$ of $1 \mathrm{~T}$.

More information about the fabrication and characterization of the $\mathrm{NdFeB}$ films will be detailed elsewhere.

\section{Magnetic force calculations}

Although a magnetic layer was deposited everywhere on the chip, only the magnetic layer sputtered on the top of the pillars (Fig. 1) provides a useful force for diamagnetic trapping. The magnetic layer deposited within the grooves can be neglected for two reasons:

- Sputtering being a highly directional deposition process, film deposition in high aspect ratio grooves is limited because of shadowing effects.

- The depth of the grooves $(90 \mu \mathrm{m})$ is 3 times the thickness of the top magnetic layer $(30 \mu \mathrm{m})$. As a result, the bottom layer is far away from the working zone and thus does not contribute to the magnetic force.

For easier computation of the magnetic force, the top magnetic layers were assumed to have the same lateral dimensions as the underlying patterned silicon.

Two designs of magnetic chips were investigated: an array of square micromagnets (Fig. 2A) and a continuous magnetic film containing a honeycomb-like array of circular holes (Fig. 2B). Due to the magnetic material's high coercivity (far higher than the field produced by the micromagnets), no demagnetizing effects were taken into account in the calculations. For ease in modeling, the second array type was computed as the sum of one large square magnet and an array of cylindrical magnets of opposite magnetization (Fig. 2B). Thanks to this simple geometry, computations could be done analytically with the CADES framework. ${ }^{29}$

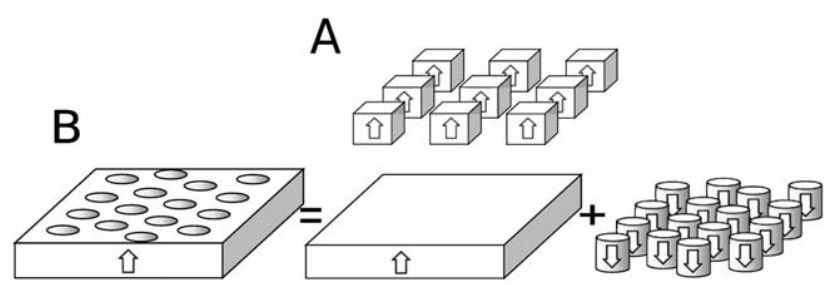

Fig. 2 Computational schemes of the micromagnet arrays: (A) square magnets, (B) honeycomb-like circular magnetic holes. The second case was computed by adding a planar magnetic layer and cylindrical magnets having an opposite magnetization direction.

\section{Experimental device}

The $8 \mathrm{~mm}$ side magnetic chip was placed in a square aluminium chamber filled with the biological buffer described hereafter. To avoid local heating of the buffer by the microscope light which can create convective flows, the temperature within the chamber was controlled by a thermoelectric Peltier device HT6-12-40 from Melcor (Trenton, USA). In addition, a quartz cover slip was used as a lid to encapsulate the chamber and to thermally insulate and flatten the water/air interface. As the substrate is not transparent, suspended cells were imaged from above using an epifluorescence microscope (Olympus BX-41) and a 460-495 nm excitation/510 $550 \mathrm{~nm}$ emission cube for acridine orange fluorescence detection.

\section{Cell culture and viability}

Jurkat lymphocyte cells were maintained in suspension in $75 \mathrm{~cm}^{2}$ flasks at a concentration of $2 \times 10^{5}$ cells $\mathrm{mL}^{-1}$ in $10 \mathrm{~mL}$ growth medium consisting of RPMI (Roswell Park Memorial Institute medium, GIBCO BRL) supplemented with $10 \%$ of Fetal Calf Serum (FCS) and $1 \%$ of Penicillin/Streptomycin (P/S). Cells were cultured at $37{ }^{\circ} \mathrm{C}$, in humidified atmosphere with $5 \% \mathrm{CO}_{2}$. Before each experiment, the cells were labelled with acridine orange (Biostatis Limited, Shepshed, UK) by suspending them for $15 \mathrm{~min}$ in a $10 \mathrm{mg} \mathrm{L}^{-1}$ acridine orange solution. After two washes, gadolinium $(\mathrm{Gd})$ based CA was added to the solution.

Viability and proliferation of Jurkat cells were monitored in the presence of 3 different Gd-based contrast agents which have comparable magnetic properties $\left(\chi_{\mathrm{v}} \approx 160 \times 10^{-6}\right.$ SI measured for Gd-DOTA with a Superconducting Quantum Interference Device, SQUID):

- Gadobenate dimeglumine (Gd-BOPTA; trade name: MultiHance) from Bracco Diagnostics (Milano, Italy). ${ }^{30}$

- Gadoterate meglumine (Gd-DOTA; trade name: Dotarem) from Guerbet (Aulnay-sous-Bois, France). ${ }^{31}$

- Gadoteridol (Gd-HP-DO3A; trade name: ProHance) from Bracco Diagnostics. ${ }^{32}$

The contrast agents were directly diluted in the cell culture medium. Cell viability was assayed regularly during an exposure of 2 days for each of the three CAs. Following this, cell recovery was plotted for 3 days after an exposure of 3 hours to the least toxic CA. In both cases, cells were washed twice, diluted twice and labelled by a Live and Dead test (Guava ViaCount Reagent) at fixed times. Cell viability was counted in triplicate using the flow cytometry system EasyCyte from Guava Technologies (San Francisco, USA).

\section{Results and discussion}

\section{Cell proliferation and viability}

As Gd-based CAs are currently used in Magnetic Resonance Imaging for medical purposes, their toxicity has already been widely investigated in vivo. ${ }^{33,34}$ However, their cytotoxicity remains little studied. As far as we know, cytotoxicities of GdDOTA and gadodiamide (Gd-DTPA-BMA) have been studied for neutrophiles. ${ }^{35,36}$ Gadopentetate dimeglubine (Gd-DTPA) cytotoxicity on fibroblast and yeast cells was briefly overviewed by Winkleman et al. on the occasion of the first diamagnetic trapping experiment description. ${ }^{26}$ More recently, a study 
comparing the cytotoxicity of several Gd-based CAs and iodinated radiographic contrast media (RCM) was carried out on kidney cells (LLC PK1). ${ }^{37}$ Because of the CA chemical complexity, cytotoxicity is poorly understood at low and high concentrations. Thus, cytotoxicity cannot be reliably extrapolated to different cell lines. For this reason, we investigated the viability and proliferation of Jurkat cells in the presence of GdBOPTA, Gd-DOTA and Gd-HP-DO3A at concentrations varying from 10 to $100 \mathrm{mM}$ (Fig. 3).

Gd-BOPTA is the most toxic contrast agent among the three tested. At high concentrations (above $85 \mathrm{mM}$ ), half of the Jurkat population dies on the first day (Fig. 3A). The second day, the cell population tends to slightly increase. For intermediate concentrations (from 25 to $50 \mathrm{mM}$ ), the proliferation is reduced on the first day and then the cell population decreases on the second day (Fig. 3A), which is in close agreement with the viability curve (Fig. 3B). Using the viability data in Fig. 3B, we speculate that high Gd-BOPTA concentrations produce cell necrosis. ${ }^{37}$ Intermediate concentrations may initiate apoptosis, thereby explaining the one day delay after Gd-BOPTA incubation. Finally, with a concentration of $10 \mathrm{mM}$ of Gd-BOPTA, cell proliferation is slowed down compared to the reference condition (0 mM), but cells do not die.

Similar to the study of LLC-PK1 cytotoxicity, ${ }^{37}$ Gd-DOTA cytotoxic effects on Jurkat cells are weaker than those from GdBOPTA incubation. At $100 \mathrm{mM}$ and $85 \mathrm{mM}$, cell proliferation decreases or slightly increases, respectively (Fig. 3C) with a death ratio of $30-40 \%$ after 2 days (Fig. 3D). At concentrations below $50 \mathrm{mM}$, cells proliferate almost normally with excellent cell viability ratios.

Finally, cytotoxic effects due to Gd-HP-DO3A are the lowest of the $3 \mathrm{CAs}$ as Jurkat cells continue to proliferate even at high concentrations of this CA (Fig. 3E and F). At concentrations higher than $50 \mathrm{mM}$, a slight reduction of cell viability can be noticed. Below $25 \mathrm{mM}$, no noticeable effects can be detected.

Toxicity of Gd-contrast agents is generally explained either by the dissociation of the chelate complex and the transmetallation kinetics, ${ }^{38}$ or by osmolality of the chemical compounds in the solution (osmolality is proportional to the number of particles in solution and inversely proportional to the molecular weight). ${ }^{37,39}$ Transmetallation results in $\mathrm{Gd}^{3+}$ exchange with another ion such as $\mathrm{Ca}^{2+}$ within the chelate complex. $\mathrm{As}^{2} \mathrm{Gd}^{3+}$ has approximately the same size as $\mathrm{Ca}^{2+}$, free $\mathrm{Gd}^{3+}$ can block calcium channels in the cell membrane. Even at extremely low concentrations, free $\mathrm{Gd}^{3+}$ is very toxic.

Gd-BOPTA is a linear chelate contrary to Gd-DOTA and GdHP-DO3A which are macrocyclic chelates. In general, linear chelates are less stable than macrocyclic ones. ${ }^{40}$ Their dissociation kinetics are much faster and they might have a thermodynamic state more favourable to transmetallation. At high concentrations, transmetallation might occur since according to Löwe et al., at $50 \mathrm{mM}, 10 \%$ of the $\mathrm{Ca}^{2+}$ ions interact with
A

C
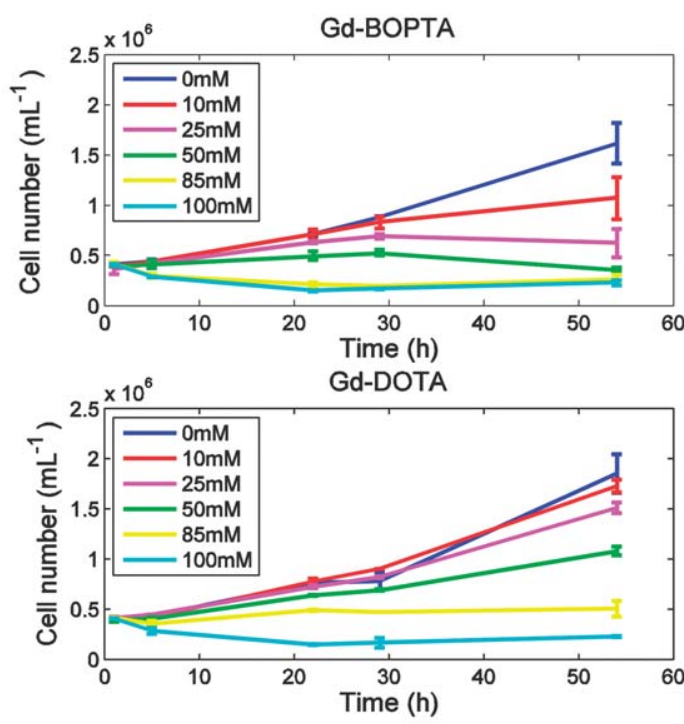

E

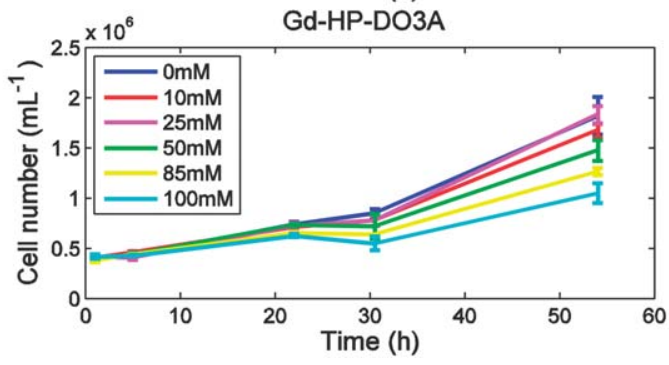

B

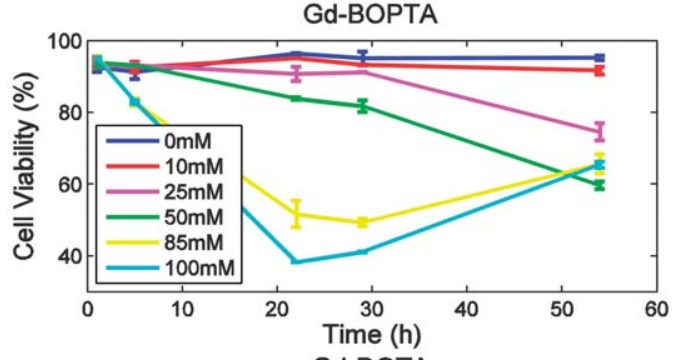

$\mathrm{D}$

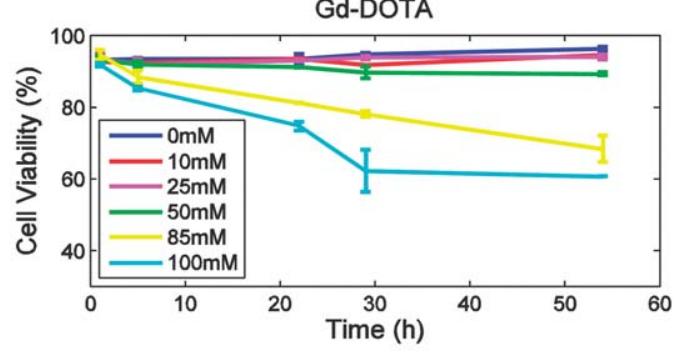

Gd-HP-DO3A

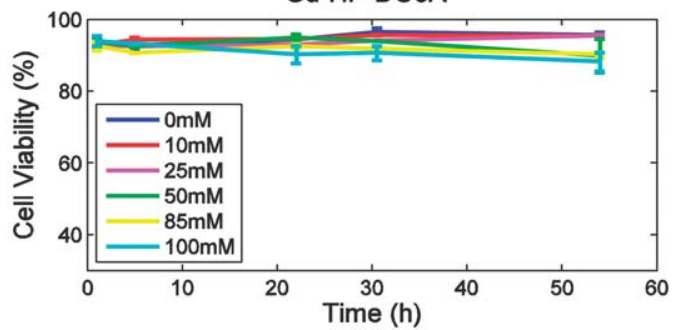

Fig. 3 Cell proliferation (A, C, E) and viability (B, D, F) of Jurkat cells in the presence of the contrast agents Gd-BOPTA (A and B), Gd-DOTA (C and D) and Gd-HP-DO3A (E and F) in concentrations varying between 10 and $100 \mathrm{mM}$. Scaling is identical for all graphs of a given column in order to facilitate comparisons. The Gd-HP-DO3A shows the smallest deviation from the reference condition $(0 \mathrm{mM})$. 
Gd-BOPTA.$^{41}$ Furthermore, although Gd-DOTA is chemically more stable than Gd-HP-DO3A, ${ }^{38}$ it is also more toxic (Fig. 3CF). $\mathrm{Gd}^{3+}$ release is thus not sufficient to explain the higher cytotoxicity of Gd-DOTA compared to Gd-HP-DO3A.

Interestingly, Gd-BOPTA ${ }^{2-}$, Gd-DOTA ${ }^{-}$and Gd-HP-DO3A have 2, 1 and 0 charges, respectively. Due to electroneutrality, each charge of the chelate complex must be balanced by a monovalent counter-ion. The number of compounds carried in the structural unit of Gd-BOPTA and Gd-DOTA contrast agent solutions is thus 3 and 2 times higher than in the Gd-HP-DO3A solution. As a result, the osmotic pressures of Gd-BOPTA and Gd-DOTA solutions are about 3 times and 2 times higher than that of Gd-HP-DO3A solution, respectively: at $0.5 \mathrm{M}$ their respective osmolalities are 1.97, 1.35 and $0.63 \mathrm{Osm} \mathrm{kg}^{-1}$, which are all higher than that of the physiological medium ( $0.3 \mathrm{Osm}$ $\left.\mathrm{kg}^{-1}\right){ }^{38}$ Osmotic pressure may thus play a role in the classification of CAs according to their cytotoxicity toward Jurkat cells observed in Fig. 3. However, CA cytotoxicity due to osmotic stress is still debated. Heinrich et al. did observe that the CA with the highest osmolality demonstrates the greatest cytotoxicity. However, they also noticed that mannitol based hypertonic solution is less cytotoxic than the same $\mathrm{CA}$ at intermediate concentrations (i.e. 31.25 and $62.50 \mathrm{mM}$ ). ${ }^{37}$ Consequently, neither transmetallation nor osmolality can explain independently the observed toxicity. However, combined together these two phenomena might explain such toxicity.

In the High Content Analysis (HCA) framework, the cells in suspension above the micro-magnet array device should only be temporarily exposed to the contrast agent, i.e. only the few tens of minutes needed for cell sedimentation and optical characterization of the whole cell population by automated microscopy. In order to assess the cytotoxic effect on Jurkat cell growth after a relatively brief exposure to the Gd-HP-DO3A contrast agent, Jurkat cells were incubated for three hours with Gd-HP-DO3A, then washed and incubated in their standard growth medium for 64 hours. Cells perfectly recovered from their $3 \mathrm{~h}$ exposure for CA concentrations up to $100 \mathrm{mM}$ (Fig. 4). This excellent recovery shows that no long-term effects are associated with GdHP-DO3A exposure. We thus consider that Jurkat cells are minimally affected by Gd-HP-DO3A and can potentially be recovered from the cell array device for further cell growth, cloning, genotyping or phenotyping characterizations. This contrast agent was thus selected for the experiments described hereafter.

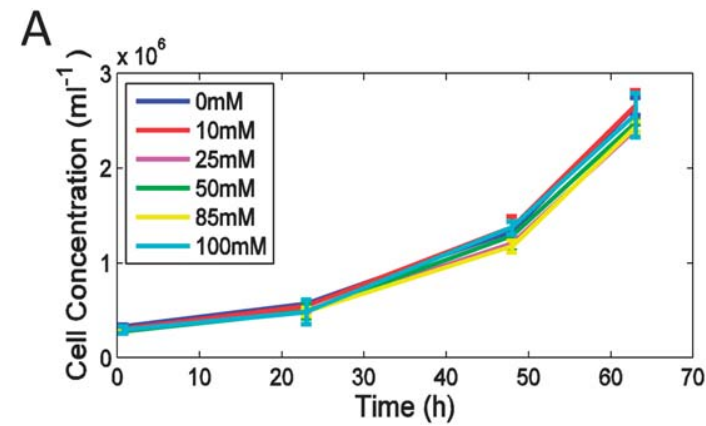

Although the cytotoxicity has been studied in an off-chip environment, no toxicity effect is expected from the magnetic field $(<1 \mathrm{~T}),{ }^{42,43}$ nor the field gradients which apply tension forces similar to the weight of the cell $(\sim 1 \mathrm{pN})$ (i.e. at least 3 orders of magnitude less than the mechanical force exerted on the cell membrane by the cytoskeleton $(1-10 \mathrm{nN}))^{44}$

\section{Cell arraying on square shape micro-magnet array}

Simulation of Jurkat cell arraying. The magnetic chips have been designed so as to provide a magnetic gradient high enough to capture sedimenting cells, even at low CA concentrations $(<10 \mathrm{mM})$ i.e. with a very weak susceptibility contrast between the cells and their medium. Fig. 5 plots the analytical computation of potential energy (magnetic and gravitation) of a Jurkat cell trapped above the micromagnet array made of $20 \times 20 \mu \mathrm{m}$ wide and $30 \mu \mathrm{m}$ thick rectangular parallelepiped magnets. For $10 \mathrm{mM}$ of Gd-HP-DO3A, the potential energy minima, corresponding to magnetic virtual traps for cells, are located $8 \mu \mathrm{m}$ above the top magnetic layer, and just between two adjacent micromagnets (Fig. 5A and B).

The impact of the CA concentration on the magnetic trap is calculated so as to evaluate the minimum concentration required for efficient diamagnetic trapping. As the molar density of $\mathrm{Gd}-$ HP-DO3A (1.274 $\mathrm{g}^{\text {per }}\left(\mathrm{mol} \mathrm{L}^{-1}\right)$ i.e. $1.005 \mathrm{~g} \mathrm{~mL}^{-1}$ for $10 \mathrm{mM}$ ) cannot be neglected compared to that of the cell $(\sim 1.080 \mathrm{~g}$ $\left.\mathrm{mL}^{-1}\right),{ }^{45} \mathrm{CA}$ density and susceptibility are both taken into account in the calculations of the diamagnetic trapping. Between $1.5 \mathrm{mM}$ and $10 \mathrm{mM}$ of CA, the depth of the magnetic well (i.e. the trapping stability) increases with the CA concentration (Fig. 5C and D) due to the corresponding increase of susceptibility contrast. The increase of the levitation height with the CA concentration (Fig. 5C) is mainly explained by the increase in medium density. Below $1.5 \mathrm{mM}$ of CA, no trapping is possible (Fig. 5C) as the magnetic force is too small to compensate for the apparent weight of the cell.

Energy is displayed in $k_{\mathrm{B}} T$ per cell so that energy trapping can be easily compared to the Brownian motion experienced by a cell. The large value of the energy well $\left(10^{2}-10^{4} k_{\mathrm{B}} T\right.$; insets of Fig. 5C and $\mathrm{D}$ ) shows that diffusion does not disturb diamagnetic trapping even at $1.5 \mathrm{mM}$ of CA. However, at the micrometre scale, the laminar drag force, which depends on the radius $r$ of the cell, overcomes the volume diamagnetic force. For this reason, diamagnetic trapping remains sensitive to any convective flows.

B

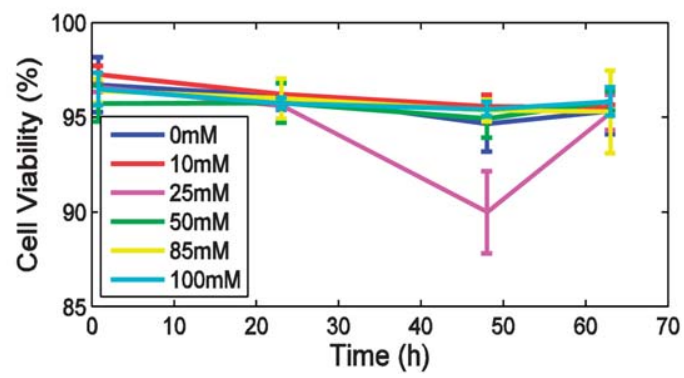

Fig. 4 Jurkat cell recovery after an exposure of 3 hours to Gd-HP-DO3A. (A) Cell proliferation. (B) Cell viability. The measurement discrepancy at $49 \mathrm{~h}$ for the $25 \mathrm{mM}$ concentration is considered to be an artefact. 

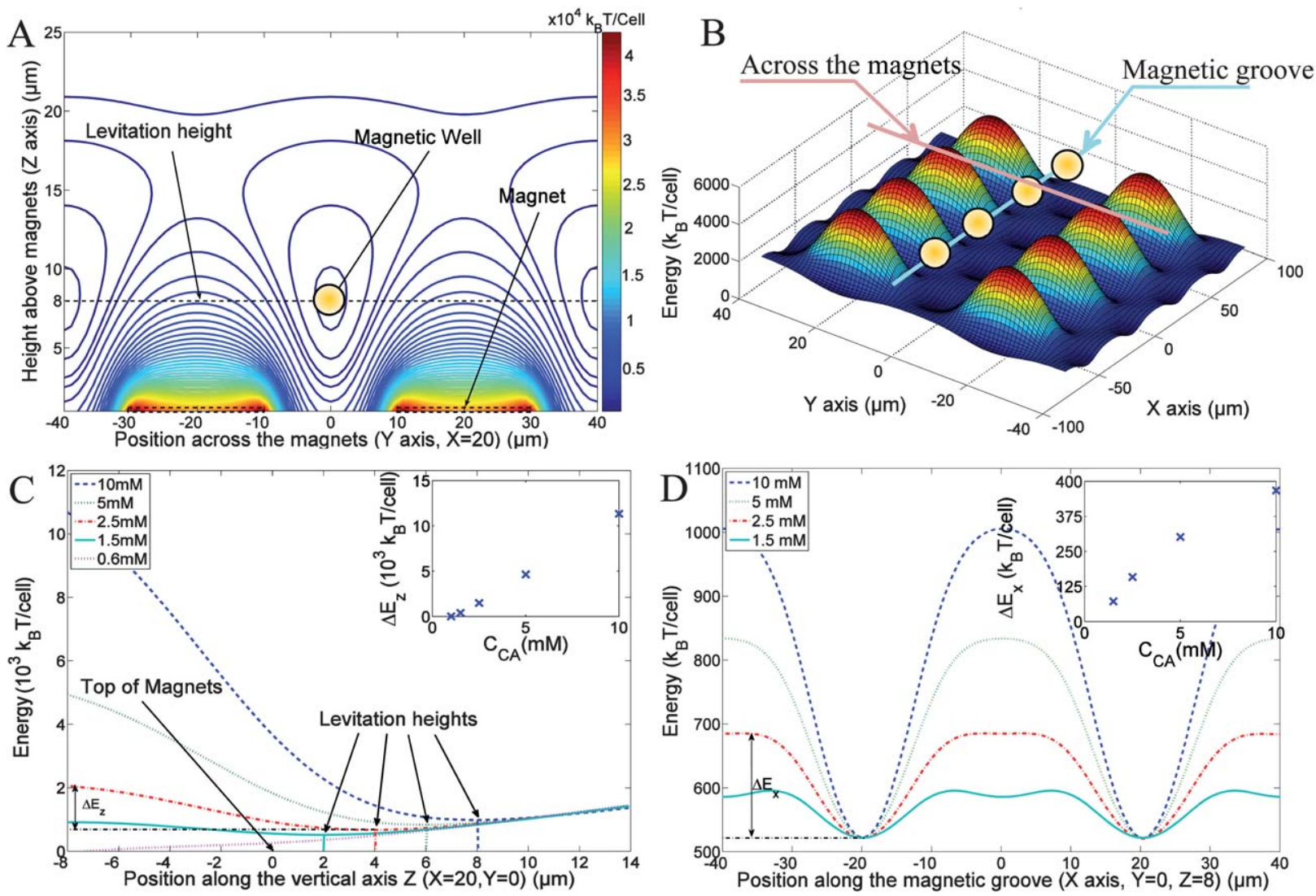

Fig. 5 Analytical simulations of diamagnetic cell trapping above an array of square micro-magnets. (A) Sideview of the potential energy isovalues across the magnet for $10 \mathrm{mM} \mathrm{CA}$. The cell in suspension (circle) is trapped in the magnetic well between the square shape magnets. (B) Magnetic well map in the levitation plane for $10 \mathrm{mM} \mathrm{CA}$. Cells are localised between the edges of the magnets at $z \approx 8 \mu \mathrm{m}$ above the top of the magnets. (C and D) Evolution of the magnetic well position according to the concentration of the CA. Two components are displayed: (C) the vertical component between two magnets; (D) the horizontal component along a magnetic groove between two magnets. Insets: evolution of the magnetic well depth according to the CA concentration. The diamagnetic trap remains stable down to a CA concentration of $1.5 \mathrm{mM}$.

The simple use of a cover slip above the cell array device to thermally insulate the water/air interface solves this issue.

In a general manner, the diamagnetic force can hardly hold cells in laminar flows. Rinsing, staining or exposure of cells to drugs after diamagnetic cell arraying appear difficult to achieve. For applications involving these operations on the chip, cells would rather be arrayed by dielectrophoresis for flows up to $5 \mu \mathrm{m}$ $\mathrm{s}^{-1},{ }^{16}$ or by acoustic tweezers for flows up to few tens of $\mu \mathrm{m}$ per second. ${ }^{17}$ Diamagnetic cell arraying is thus more adequate to optical characterization of cells at endpoints of HCA experiments or transiently before a recovery of the whole cell population. An exposure of cells to a gradient of drug concentration gently produced by molecular diffusion could also be achievable.

Experimental results of Jurkat cell arraying. The $8 \times 8 \mathrm{~mm}$ cell array device (Fig. 6A) was placed at the bottom of a $270 \mu \mathrm{L}$ chamber. Then, the cell suspension at a concentration of $2 \times 10^{5}$ cells $\mathrm{mL}^{-1}$ was poured onto the chip. As soon as the solution wetted the chip, cells sedimented. When approaching the magnetic layer, the diamagnetic force deflected their trajectories. In less than $10 \mathrm{~min}$, all cells were trapped between the edges of the square shape magnets, a few micrometres above the top of the magnetic layer.
Concentrations of Gd-HP-DO3A from $10 \mathrm{mM}$ down to $0.66 \mathrm{mM}$ have been tested. Between $10 \mathrm{mM}$ and $1.5 \mathrm{mM}$ of CA, most of the cells were correctly trapped in diamagnetic wells. In

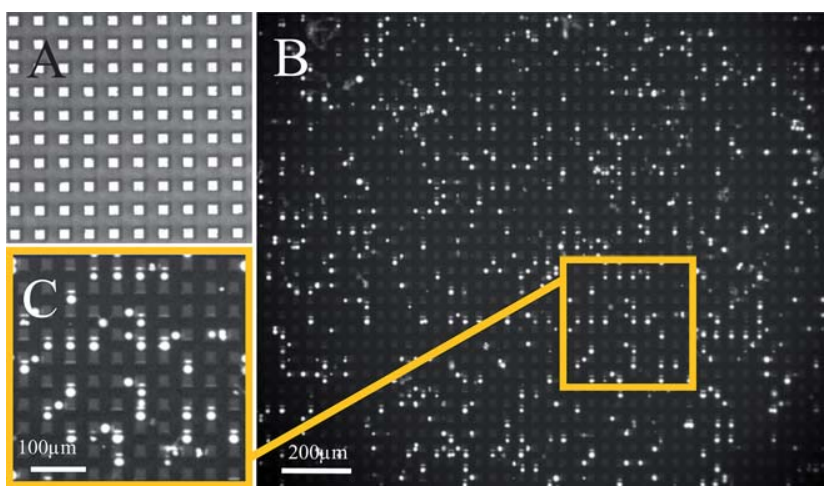

Fig. 6 Arraying of Jurkat cells between $20 \times 20 \mu \mathrm{m}$ square shape micromagnets in $1.5 \mathrm{mM}$ of Gd-HP-DO3A. (A) Top view of the micromagnet array (white squares). (B) $1.5 \times 1.3 \mathrm{~mm}$ top view of acridine orange labelled fluorescent cells (white circular spots) trapped between the square shape micromagnets (dark squares), imaged using fluorescence microscopy. (C) Enlargement of the area shown in (B). 

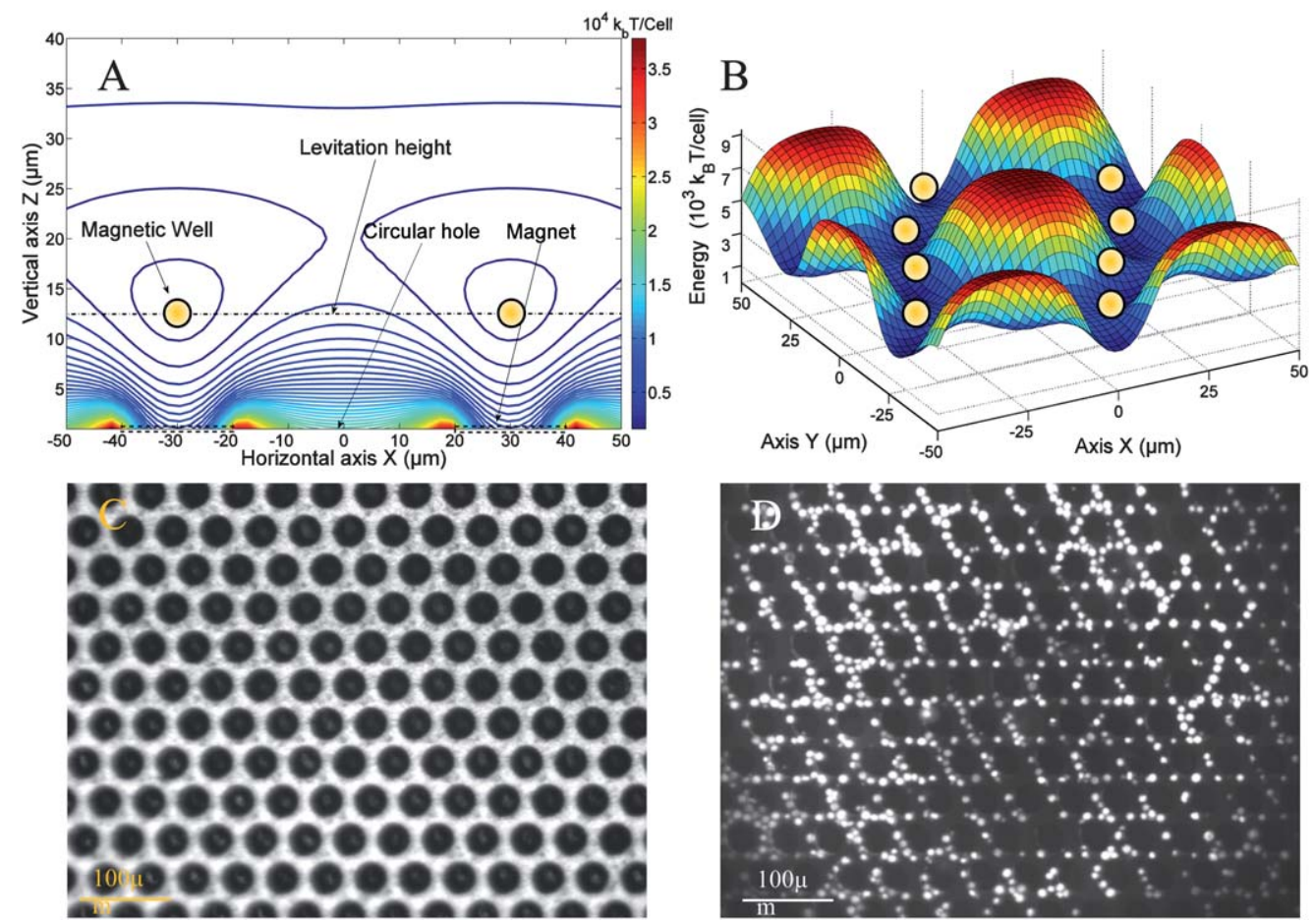

Fig. 7 Arraying of Jurkat cells trapped above a honeycomb-like micro-magnet array. (A) Sideview of the potential energy isovalues above the magnetic layer. Magnetic wells are designed between the holes, i.e. above the magnetic layer, at a height of $\sim 12.5 \mu \mathrm{m}$. (B) Magnetic well map in the levitation plane $(z \approx 13 \mu \mathrm{m})$. (C) Top view of the permanent magnet (white surface) containing $40 \mu \mathrm{m}$-large holes (black circles). (D) Top view of the acridine orange labelled fluorescent cells (white circular spots) trapped above the magnetic layer with $10 \mathrm{mM}$ Gd-HP-DO3A.

Fig. $6 \mathrm{~B}$, at $1.5 \mathrm{mM}$ of Gd-HP-DO3A, less than $1 \%$ of cells were stuck on the chip and less than $10 \%$ were trapped in the same well. The magnetic surface behaving as a "diamagnetophobic surface", almost no cells sedimented onto the chip. However, at $1 \mathrm{mM}$ and $0.66 \mathrm{mM}$ of Gd-HP-DO3A, the trapping quality decreased and positioning was less accurate. Cells were either trapped between magnets or at the intersection of the grooves where they may eventually sediment. Finally, without any CA, no diamagnetic trapping was observed. In close agreement with the simulations, the minimal concentration of CA for trapping cells above the array of square micromagnets is around $1.5 \mathrm{mM}$. This is more than 13 times lower than formerly reported works. $^{26,46}$ It corresponds to a susceptibility contrast $\Delta \chi=$ $-0.8 \times 10^{-6} \mathrm{SI}$ which is ten times smaller than water $\left(\chi_{\text {water }}=\right.$ $-9 \times 10^{-6} \mathrm{SI}$ ), thereby confirming that the cell medium need not be paramagnetic but only less diamagnetic than the cells. ${ }^{46}$

Fluorescent light emitted by labelled cells seems to reflect off one of the edges of the magnets (Fig. 6B and C), most likely due to a dissymmetry of the edge of the magnets caused by a slight inclination of the wafer during the sputtering of $\mathrm{NdFeB}$. This issue could be overcome by the use of planar magnets array ${ }^{47}$ or by using an anti-reflective coating (e.g. $50 \mathrm{~nm} \mathrm{SiN}$ ).

In order to evaluate the influence of temperature on diamagnetic trapping, cells were suspended at different temperatures, at $2.5^{\circ} \mathrm{C}, 10{ }^{\circ} \mathrm{C}, 20^{\circ} \mathrm{C}$ and $37^{\circ} \mathrm{C}$, continuously for up to $4 \mathrm{~h}$. For the whole temperature range, cells remain stably trapped during this period. Slight movements within the trap were observed at each temperature, thereby confirming that the cells are not stuck and that the trapping energy in the magnetic traps exceeds the thermal energy. However, when the cover slip was removed, thermal convection due to microscope illumination heating dragged the cells horizontally out of their trap.

\section{Cell aligned on honeycomb micro-magnet array}

Trapping cells above the magnetic material rather than in the space between magnetic sections was achieved using a honeycomb array composed of $40 \mu \mathrm{m}$ diameter circular holes in the magnet layer, separated by $20 \mu \mathrm{m}$ (Fig. 7A and C).

Analytical computations of the side view of the magnetic and gravitational potentials of a cell show a trapping height of $12.5 \mu \mathrm{m}$ above the magnets (Fig. 7A). Fig. 7B displays the energy map of the cell in the levitation plane, thereby confirming that the cells are always trapped above the magnets.

Experimental observations confirm computational results. Jurkat cells at a concentration of $2 \times 10^{5}$ cells $\mathrm{mL}^{-1}$ suspended in the RPMI medium containing $10 \mathrm{mM}$ Gd-HP-DO3A were poured onto the magnet array. While cells sedimented toward the micro-magnet array, they aligned between the holes above the magnet layer (Fig. 7D). No fluorescence was observed inside the holes, indicating that the cell population sedimented only above the magnet layer. Cells moved slightly above the magnet, thereby confirming that they are trapped without contact. A trapping height of $13 \mu \mathrm{m}$ was measured between the cells and the top of the magnet layer by focusing the microscope successively onto the cells and the magnet layer.

The presence of holes inside the magnet layer produced a strong repulsion, in the $10^{3} k_{\mathrm{B}} T$ range, of the cells towards the 
magnet layer (Fig. 7B). In a similar manner to the array of square micro-magnets, the honeycomb micro-magnet array acts as a "diamagnetophobic" surface for the cells, as the cells in suspension were repelled by the surface onto which they sedimented. The repulsion was sufficient to maintain the Jurkat cells about $13 \mu \mathrm{m}$ above the magnet layer, i.e. more than twice the radius of a Jurkat cell ( $r \approx 5 \mu \mathrm{m})$.

Jurkat cells have a density between 1.080 and $1.090 \mathrm{~g} \mathrm{~mL}^{-1}$. $^{8}$ Hence theoretically, the difference of levitation height among Jurkat cells could reach a maximum of $1 \mu \mathrm{m}$, for CA concentrations between $1.5 \mathrm{mM}$ and $10 \mathrm{mM}$. This difference is negligible compared to the depth of field $d_{z} \approx 8 \mu \mathrm{m}$ of the $10 \times$ objective (NA $=0.30)$ used to acquire our images. ${ }^{49}$ The slight focus difference, which can be noticed at least in Fig. 7D, would rather be due to a slight tilt or magnetic inhomogeneity of the chip. However, a bigger difference of density can be observed between two different cell lines. Among circulating cells, the density could vary from $1.040 \mathrm{~g} \mathrm{~mL}^{-1}$ (thrombocyte) to $1.100 \mathrm{~g} \mathrm{~mL}^{-1}$ (erythrocyte). ${ }^{48}$ The difference of trapping height is estimated to be around $5 \mu \mathrm{m}$, which is still included in the depth of field of the $10 \times$ objective.

\section{Conclusions}

High quality $\mathrm{NdFeB}$ micro-magnet arrays $\left(J_{\mathrm{r}} \approx 1 \mathrm{~T}\right)$ have been microfabricated to provide highly inhomogeneous magnetic fields intended for diamagnetic trapping and arraying of nonadherent cells. For the diamagnetic force to be repulsive, the medium needs to be less diamagnetic than the cells. To achieve this, a paramagnetic contrast agent was added to the solution. The biocompatibility of three contrast agents was compared and discussed. According to proliferation and viability tests, Gd-HPDO3A is far more biocompatible with Jurkat cells than Gd-BOPTA or Gd-DOTA. In agreement with analytical simulations, concentrations of Gd-HP-DO3A as low as $1.5 \mathrm{mM}$ allow the arraying of Jurkat cells a few micrometres above the surface of the micromagnet array without any toxic effect on the cells. Two types of micromagnet patterns were used to demonstrate arraying either above the gap between square micromagnets or above the magnet material in a honeycomb-like configuration. Stable cell trapping has been observed for more than 4 hours at temperatures up to $37^{\circ} \mathrm{C}$, demonstrating that trapping remains stable in spite of the cells' thermal motion.

Contactless diamagnetic cell arraying using a low concentration of Gd-HP-DO3A provides a biocompatible, simple to use technique for the efficient and rapid ordering of cells in suspension. The cell array device needs no power supply and thus the cells are not submitted to any Joule heating. This "diamagnetophobic surface" appears promising for handling and arraying cells as well as any other diamagnetic objects in suspension. Rapid, contactless arraying of cells could be valuable in High Content Analysis (HCA) experiments to characterize the cells after their exposure to a specific drug or environment, or for briefly observing the cell population before recovery and subsequent out-of-the-chip steps. Gentle exposure of the arrayed cells to a drug gradient produced by molecular diffusion is also envisioned. This precise cell positioning technique could allow the study of the cell-cell interactions, cell motility and eventually cell differentiation.

\section{Acknowledgements}

The authors thank Delphine Constantin, Irène Pheng and Stéphane Litaudon (CIME, Grenoble) for their assistance in substrate patterning. The authors also thank Christian Jeandey and Jean-François Jacquot (CEA, Grenoble) for the susceptibility measurements and Jean-Marc Constans (CHU, Caen) for providing the contrast agents. Funding was provided by Grenoble INP and the Rhône-Alpes region.

\section{Notes and references}

1 D. Di Carlo and L. P. Lee, Anal. Chem., 2006, 78, 7918-7925.

2 X. Gidrol, B. Fouqué, L. Ghenim, V. Haguet, N. Picollet-D'hahan and B. Schaack, Curr. Opin. Pharmacol., 2009, 9, 664-668.

3 M. Théry, V. Racine, A. Pépin, M. Piel, Y. Chen, J.-B. Sibarita and M. Bornens, Nat. Cell Biol., 2005, 7, 947-953.

4 D. Di Carlo, L. Y. Wu and L. P. Lee, Lab Chip, 2006, 6, 1445-1449.

5 M. Tanase, E. J. Felton, D. S. Gray, A. Hultgren, C. S. Chen and D. H. Reich, Lab Chip, 2005, 5, 598-605.

6 K. Ino, M. Okochi, N. Konishi, M. Nakatochi, R. Imai, M. Shikida, A. Ito and H. Honda, Lab Chip, 2008, 8, 134-142.

7 P. Tseng, D. Di Carlo and J. W. Judy, Nano Lett., 2009, 9, 3053-3059.

8 J. R. Rettig and A. Folch, Anal. Chem., 2005, 77, 5628-5634.

9 M. Deutsch, A. Deutsch, O. Shirihai, I. Hurevich, E. Afrimzon, Y. Shafran and N. Zurgil, Lab Chip, 2006, 6, 995-1000.

10 Y. Tokimitsu, H. Kishi, S. Kondo, R. Honda, K. Tajiri, K. Motoki, T. Ozawa, S. Kadowaki, T. Obata, S. Fujiki, C. Tateno, H. Takaishi, K. Chayama, K. Yoshizato, E. Tamiya, T. Sugiyama and A. Muraguchi, Cytometry, Part A, 2007, 71, 1003-1010.

11 J. Park, M. Morgan, A. Sachs, J. Samorezov, R. Teller, Y. Shen, K. Pienta and S. Takayama, Microfluid. Nanofluid., 2010, 8, 263-268.

12 T. R. Malek, T. J. Fleming and E. K. Codias, Semin. Immunol., 1994, 6, 105-113.

13 T. Zell, W. Kivens, S.-A. Kellermann and Y. Shimizu, Immunol. Res., 1999, 20, 127-145.

14 A. Ashkin, Phys. Rev. Lett., 1970, 24, 156-159.

15 D. G. Grier, Nature, 2003, 424, 810-816.

16 N. Mittal, A. Rosenthal and J. Voldman, Lab Chip, 2007, 7, $1146-$ 1153.

17 J. Shi, D. Ahmed, X. Mao, S.-C. S. Lin, A. Lawit and T. J. Huang, Lab Chip, 2009, 9, 2890-2895.

18 H. Chetouani, C. Jeandey, V. Haguet, H. Rostaing, C. Dieppedale and G. Reyne, IEEE Trans. Magn., 2006, 42, 3557-3559.

19 C. Pigot, H. Chetouani, G. Poulin and G. Reyne, IEEE Trans. Magn., 2008, 44, 4521-4524.

20 O. Cugat, J. Delamare and G. Reyne, IEEE Trans. Magn., 2003, 39, 3607-3612.

21 N. M. Dempsey, A. Walther, F. May, D. Givord, K. Khlopkov and O. Gutfleisch, Appl. Phys. Lett., 2007, 90, 092509.

22 A. Walter, C. Marcoux, B. Desloges, R. Grechishkin, D. Givord and N. Dempsey, J. Magn. Magn. Mater., 2009, 321, 590-594.

23 E. Beaugnon and R. Tournier, Nature, 1991, 349, 470.

24 M. V. Berry and A. K. Geim, Eur. J. Phys., 1997, 18, 307-313.

25 Y. Ikezoe, N. Hirota, J. Nakagawa and K. Kitazawa, Nature, 1998, 393, 749-750.

26 A. Winkleman, K. L. Gudiksen, D. Ryan, G. M. Whitesides, D. Greenfield and M. Prentiss, Appl. Phys. Lett., 2004, 85, 2411-2413.

27 I. F. Lyuksyutov, D. G. Naugle and K. D. D. Rathnayaka, Appl. Phys. Lett., 2004, 85, 1817-1819.

28 M. Kustov, P. Laczkowski, D. Hykel, K. Hasselbach, F. DumasBouchiat, D. O'Brien, P. Kauffmann, R. Grechishkin, D. Givord, G. Reyne, O. Cugat and N. M. Dempsey, J. Appl. Phys., 2010, 108, 063914.

29 B. Delinchant, F. Wurtz, D. Magot and L. Gerbaud, Simulation, 2004, 80, 347-356.

30 M. A. Kirchin, G. P. Pirovano and A. Spinazzi, Invest. Radiol., 1998, 33, 798-809.

31 J. C. Bousquet, S. Saini, D. D. Stark, P. F. Hahn, M. Nigam, J. Wittenberg and J. T. Ferrucci, Radiology, 1988, 166, 693-698.

32 V. M. Runge, D. Y. Gelblum, M. L. Pacetti, F. Carolan and G. Heard, Radiology, 1990, 177, 393-400. 
33 F. G. Shellock and E. Kanal, J. Magn. Reson. Imaging, 1999, 10, 477484.

34 V. M. Runge, Top. Magn. Reson. Imaging, 2001, 12, 309-314.

35 J. Behra-Miellet, B. Gressier, C. Brunet, T. Dine, M. Luyckx, M. Cazin and J.-C. Cazin, Methods Find. Exp. Clin. Pharmacol., 1996, 18, 437-442.

36 J. Behra-Miellet, B. Gressier and C. Brunet, Eur. J. Pharm. Biopharm., 1995, 41, 354-360.

37 M. C. Heinrich, M. K. Kuhlmann, S. Kohlbacher, M. Scheer, A. Grgic, M. B. Heckmann and M. Uder, Radiology, 2007, 242, 425-434.

38 J.-M. Idée, M. Port, I. Raynal, M. Schaefer, S. Le Greneur and C. Corot, Fundam. Clin. Pharmacol., 2006, 20, 563-576.

39 P. Dawson, R. G. Grainger and J. Pitfield, Clin. Radiol., 1983, 34, 221-226.

40 H. Schmitt-Willich, Br. J. Radiol., 2007, 80, 581-582.

41 A. Löwe, T. Balzer and U. Hirt, Invest. Radiol., 2005, 40, 521-525.
42 J. F. Schenck, Prog. Biophys. Mol. Biol., 2005, 87, 185-204.

43 J. Miyakoshi, Sci. Technol. Adv. Mater., 2006, 7, 305-307.

44 C. S. Chen, J. Tan and J. Tien, Annu. Rev. Biomed. Eng., 2004, 6, 275 302.

45 P. Outteridge, K. Fahey and C. Lee, Aust. J. Exp. Biol. Med. Sci., 1981, 59, 143-155.

46 M. Frénéa-Robin, H. Chetouani, N. Haddour, H. Rostaing, J. Laforet, and G. Reyne, in Proc. 30th Annual International Conference of the IEEE Engineering in Medicine and Biology Society, Vancouver, Canada, Aug. 20-24, 2008, pp. 3360-3363.

47 F. Dumas-Bouchiat, L. F. Zanini, M. Kustov, N. M. Dempsey, R. Grechishkin, K. Hasselbach, J. C. Orlianges, C. Champeaux, A. Catherinot and D. Givord, Appl. Phys. Lett., 2010, 96, 102511.

48 B. E. Kellet, B. Han, D. S. Dandy and S. R. Wickramasinghe, Artif. Organs, 2004, 28, 1026-1034.

49 C. D. Meinhart, S. T. Wereley and M. H. B. Gray, Meas. Sci. Technol., 2000, 11, 809-814. 